\title{
Quantitative Magnetic Resonance-Derived Biomarkers as Predictors of Function and Histotype in Adenohypophyseal Tumours
}

\author{
Daniel Lewis ${ }^{a, b, c}$ Federico Roncarolia, c, d, e Tara Kearneyd, e \\ David John Coope ${ }^{a, c, d, e}$ Kanna Gnanalingham ${ }^{a, c}$
}

\begin{abstract}
aDepartment of Neurosurgery, Manchester Centre for Clinical Neurosciences, Salford Royal NHS Foundation Trust, Manchester Academic Health Science Centre, Manchester, UK; ${ }^{b}$ Division of Informatics, Imaging and Data Sciences, Wolfson Molecular Imaging Centre (WMIC), University of Manchester, Manchester, UK; ' Geoffrey Jefferson Brain Research Centre, University of Manchester, Manchester, UK; dDivision of Neuroscience and Experimental Psychology, School of Biological Sciences, Faculty of Biology, Medicine and Health, University of Manchester, Manchester, UK; 'Department of Endocrinology, Salford Royal NHS Foundation Trust, Manchester Academic Health Science Centre, Manchester, UK
\end{abstract}

\section{Keywords}

Adenohypophyseal tumours · Biomarker · Growth hormone $\cdot$ Magnetic resonance imaging $\cdot$ Non-functioning gonadotropinoma $\cdot$ Pituitary tumours $\cdot$ Prolactin

\begin{abstract}
Introduction: Magnetic resonance imaging (MRI) is the main modality to diagnose adenohypophyseal tumours, while biochemical assessment of pituitary hormones allows for their functional classification. In this retrospective exploratory cohort study, we investigated if quantitative differences in tumour MR signal intensity (SI) could be utilized to predict the function and histotype. Methods: Clinically acquired pretreatment MRI images were retrospectively analysed in 67 clinically non-functioning gonadotropinomas (NFG), 38 somatotropinomas, and 16 medically treated giant macroprolactinomas. Mean T1- and T2-weighted SI values were determined for each tumour and normalized against either centrum semiovale white matter or CSF to derive relative $\mathrm{T} 1 \mathrm{~W}$ and $\mathrm{T} 2 \mathrm{~W}$ SI values and the relative tumour T2/T1 SI ratio. Inter-group differences in quantitative MR parameters were compared, and the power of each parameter to discriminate tumour type and subtype was assessed using the area under the receiver operator characteristic curve (AUROC). In resect-
\end{abstract}

ed somatotropinomas, the relationship between tumour granulation status, relative MR SI values, and biochemical data was also compared. Results: Compared to somatotropinomas, NFG and macroprolactinomas displayed higher relative T2W SI $(p<0.001)$ and higher relative tumour T2/T1 SI ratio values ( $p<0.001$, ANOVA). Compared to intermediate/densely granulated tumours, sparsely granulated somatotropinomas were larger $(p=0.006$, Mann-Whitney $U$ test), had higher relative T2W SI ( $p \leq 0.005)$, and higher relative tumour T2/T1 SI ratios ( $p \leq 0.001,2$-tailed $t$ test). Relative tumour T2W SI values and relative tumour T2/T1 ratio values demonstrated good discriminatory power in differentiating NFG from somatotropinoma (AUROC $=0.87-0.94$ ) and predicting somatotropinoma subtypes (AUROC $=0.87-0.95$ ). Conclusion: Quantitative SI-based MR parameters derived using clinical acquisition MRI protocols may help non-invasively discriminate the functional status of adenohypophyseal tumours and the histological subtype of somatotropinomas.

(c) 2021 The Author(s).

Published by S. Karger AG, Basel

A portion of this work was presented as an oral abstract at the Spring 2019 Joint Meeting of the Society of British Neurological Surgeons and the European Association of Neuroscience Nurses, Manchester, UK.
C) 2021 The Author(s).

Published by S. Karger AG, Basel

This is an Open Access article licensed under the Creative Commons Attribution-NonCommercial-4.0 International License (CC BY-NC) (http://www.karger.com/Services/OpenAccessLicense), applicable to the online version of the article only. Usage and distribution for commercial purposes requires written permission.
Correspondence to:

Daniel Lewis, daniel.lewis@srft.nhs.uk 


\section{Introduction}

Tumours of adenohypophyseal cells account for around $10 \%$ of all intracranial neoplasms and approximately $90 \%$ of all sellar tumours [1]. About $60 \%$ are clinically functioning causing signs and symptoms due to hormone hypersecretion [2]. Adenohypophyseal tumours are classified by histotype based on their immunoprofile and by hormone secretion, size, and extension. Magnetic resonance imaging (MRI) and assessment of endocrine function are the "gold standard" for preoperative assessment of sellar tumours [3]. Lactotroph and some growth hormone $(\mathrm{GH})$-secreting tumours can respond to medical therapy [4]. Surgery is the treatment of choice for clinically non-functioning tumours [5].

The role of MRI in predicting the endocrine status of adenohypophyseal tumours is not clear, with studies utilizing both qualitative and quantitative methods to assess MRI signal characteristics. Most reports have qualitatively assessed differences in MR characteristics between tumour types and subtypes by visual inspection, categorizing tumours as hypo-, iso-, or hyperintense relative to either normal brain or the pituitary gland [6-10]. Such qualitative methods are quick and readily accessible to the expert reader but they lack standardization and reproducibility as they are subject to inter-observer variability [11]. Some studies have proposed the use of T1-weighted and T2-weighted signal intensity (SI) values normalized to an internal reference region $[12,13]$. In an early low field $(0.5 \mathrm{~T})$ MRI study by Lundin et al. [12], tumour T1W SI values measured from a single tumour slice and normalized against the corpus callosum, was found to be higher in functioning than in non-functional tumours. To date, however, there have been no large studies assessing quantitative differences in T1 and T2 SI-based measures between clinically non-functioning and functioning adenohypophyseal tumours.

We analysed a single-centre retrospective cohort of previously acquired MRI scans to assess whether quantitative differences in SI could predict the functional status and histological subtypes of tumours of adenohypophyseal cells. We also sought to establish amongst a cohort of functioning somatotropinomas if differences in these derived quantitative MR parameters can be used to predict subtype (i.e., densely vs. sparsely granulated) and postoperative endocrine remission.

Pre-Treatment MRI Biomarkers of

Adenohypophyseal Tumour Phenotype

\section{Methods}

\section{Patient Identification}

This was a retrospective exploratory cohort study. Ethical approval for the study had been granted by the local research ethics committee (REC Reference, 17/EE/0488). Patients were identified retrospectively from the clinical database at Salford Royal NHS Foundation Trust, Manchester, UK. The database consisted of patients with pituitary lesions who had undergone surgical treatment over a period of 10 years and patients who had undergone medical treatment for a prolactinoma over this same period.

Data from 3 cohorts of adult patients were recruited into the study: (i) patients with non-functioning gonadotropinomas (NFG) who had no biochemical or clinical evidence of hormone hypersecretion and had undergone surgery; (ii) patients with clinical and biochemical evidence of acromegaly who had undergone primary surgical resection without preoperative medical therapy; and (iii) patients with prolactinomas who had undergone medical treatment only.

\section{Inclusion/Exclusion Criteria}

The original slides were reviewed for this study by an experienced neuropathologist (FR). In an attempt to avoid mixed tumour types, only tumours expressing FSH and/or LH beta subunit were included in the non-functioning cohort. Clinically silent corticotrophinomas and silent somatotropinomas were excluded [14]. Only somatotropinoma with clinical and biochemical evidence of acromegaly as defined by the 2017 WHO classification of pituitary tumours were included [2]. On histopathology, somatotropinomas were classified as sparsely granulated, densely granulated, or intermediate type based on $\mathrm{GH}$ expression and the presence and extent of fibrous bodies seen with the immunoreaction for cytokeratin CAM5.2 [15]. Mammosomatotroph and plurihormonal tumours containing GH-positive cells and clinically causing acromegaly were also excluded. All prolactinomas at our centre are medically managed at first and only approximately $5 \%$ require surgery due to intolerance/ineffectiveness of dopamine agonists. For the group of prolactinomas, only patients with pretreatment prolactin levels $>10,000 \mathrm{mU} / \mathrm{L}$ and neuroimaging and biochemical response to dopamine agonists were included. Because tissue confirmation of the histotype was not available for these medically treated patients, the latter selection criteria aimed to minimize the inclusion of lactotroph tumours with mixed phenotypes such as lactotroph/somatrotroph and plurihormonal tumours.

To improve the accuracy of quantitative assessment of the MR images, tumours measuring $<5 \mathrm{~mm}$ in maximal dimension, tumours with significant macrocystic and/or haemorrhagic change, and tumours that were previously irradiated were excluded. Corticotrophinomas, which are typically small $(<1 \mathrm{~cm})$ and difficult to accurately delineate on pretreatment MRI, were also excluded [16].

\section{MR Image Analysis}

All patients included in this study had undergone imaging on 1 of 5 different 1.5 T MR scanners (Siemens Avanto, Siemens Aera, Phillips Intera, GE optima MR450W, and GE Signa Excite) before surgery or medical treatment. Anonymized pretreatment MR images for each patient included in the study were exported to a university workstation for further analysis. After bias correcting each image for B1 field intensity variation [17], coronal T1W, T2W, and, where available, post-contrast T1W images were co-registered using SPM12 prior to analysis [18]. 
Tumours were manually segmented on the co-registered T2W, $\mathrm{T} 1 \mathrm{~W}$, and post-contrast coronal T1W coronal images using the region of interest (ROI) tool within ITK-snap [19]. A perimetrybased method for tumour delineation and volume measurement was used as it has been reported to have superior accuracy compared to largest area or maximal axis measurements [20]. Care was taken during delineation to avoid normal structures (e.g., cavernous sinus, optic apparatus, large vessels), and the results of segmentation were reviewed and where necessary, edited by an experienced pituitary neurosurgeon (K.G.). Using the whole tumour object masks, the mean absolute T1W and T2W SI values for each tumour were derived and validated against values obtained using a multiple ROI method whereby multiple small ROIs were placed within the central part of each tumour on consecutive image slices. To assess the intra-observer repeatability of each tumour measurement, a blinded second measurement was also undertaken 1 month later for 15 tumours in each cohort.

\section{Delineation of White Matter and CSF Reference Regions}

Relative SI ratios, adjusted to the SI within each patient's normal-appearing white matter (WM) or CSF were determined. Using the circular ROI tool within ITK-SNAP [19], appropriately sized circular ROIs were placed in multiple contiguous coronal image sections within the normal-appearing WM of the centrum semiovale and within the CSF of the lateral ventricles for each patient. The size of the circular ROIs was adjusted for each individual patient so that partial volume effects from neighbouring voxels was minimized. Regions of T2 WM hyperintensity and enlarged perivascular spaces within the centrum semiovale were avoided in ROI delineation [21], along with choroid plexus and regions of flow void within the CSF [22]. The extracted mean T1W and T2W SI values for both WM and CSF reference regions were then used to determine tumour/WM and tumour/CSF T1W and T2W SI ratios. As an additional marker, the relative tumour T2/T1 SI ratios (SI Ratio-rel) were determined, where:

$$
\text { Tumour T2/T1 SI } I_{\text {Ratio-Rel }}=\frac{(\text { Tumour T2W SI/Reference ROI T2W SI })}{(\text { Tumour T1W SI/Reference ROI T1W SI })}
$$

for WM and CSF reference ROI, respectively.

In addition to the quantitative parameters described above, somatotropinomas were classified qualitatively as either hypo- or hyperintense relative to normal-appearing WM on midsagittal coronal T2-weighted images. Classification was based on visual inspection by an observer (D.L.) and verified by a second observer (K.G.). At time of classification, both observers were blinded to clinical data and the quantitative SI ratio values for each tumour.

\section{Biochemical Data and Remission Rates}

For all tumour cohorts, serum prolactin levels were analysed using the Siemens ADVIA Centaur 2-site sandwich immunoassay (Siemens Diagnostics, Tarrytown, USA) and expressed as mU/L. For somatotropinomas, preoperative baseline insulin-like growth factor-1 (IGF-1) levels and GH nadir levels during a preoperative oral glucose tolerance test were analysed [23]. Plasma IGF-1 levels were determined by chemiluminescent immunoassay (Siemens Immulite 2000 IGF-1, Deerfield, IL, USA.), expressed as percentage increase compared with the upper limit of normal [24]. GH was assayed by chemiluminescent immunoassay utilizing the Siemens Immulite $2000 \mathrm{GH}$ Assay (lower detection threshold, 0.05 $\mu \mathrm{g} / \mathrm{L}$; analytical sensitivity, $0.001 \mu \mathrm{g} / \mathrm{L})$. GH units were expressed in mass units $(\mu \mathrm{g} / \mathrm{L})[25]$.

All trans-sphenoidal surgeries were undertaken by 1 surgeon (K.G.), using an endoscopic trans-sphenoidal approach as previously described [26]. All patients with NFG and somatotropinomas underwent surgery, while those with prolactinomas were managed medically with dopamine agonists.

In somatotropinomas, surgical debulking was attempted in selected patients where the preoperative MRI scan revealed an invasive tumour with circumferential tumour extension around the carotid arteries. In all other cases, gross total resection was attempted, irrespective of tumour size. Parasellar extension or encroachment of the tumour towards the cavernous sinus in relation to the intracavernous carotid artery was classified on MRI before surgery using the Knosp grade (Grades 0-IV) [20, 27]. Post-operatively, all patients with somatotropinomas underwent testing of the GH axis (GH nadir on oral glucose tolerance test and IGF-1) at regular intervals $[23,26]$. When early testing suggested residual tumour, as also supported by findings on a repeat MR scan, further re-exploratory surgery at 2-4 weeks post-operatively was undertaken in some patients. For somatotropinomas, definition of disease remission was according to the 2010 Consensus criteria and required both a $\mathrm{GH}$ nadir of $\leq 0.4 \mu \mathrm{g} / \mathrm{L}$ on oral glucose tolerance test and a normal age- and gender-adjusted IGF-1 [28]. Borderline remission was defined as meeting only 1 of the above criteria, and these cases were considered to be not in remission [28].

\section{Statistical Analysis}

Statistical software program Stata was used to undertake all statistical analyses. Normality and homogeneity of variance for all continuous variables were assessed using the Shapiro-Wilk and Levene test, respectively. Differences in patient age, tumour size, and quantitative MR-derived parameters between the 3 tumour subgroups (NFG, somatotropinomas, and prolactinomas) were compared using a one-way ANOVA with Bonferroni correction. For non-parametric data, the Kruskal-Wallis test with adjustment for multiple comparisons was used.

For the somatotropinoma cohort, differences in tumour size, quantitative MR-derived parameters, and biochemical data were compared against somatotropinoma subtype (intermediate/ densely vs. sparsely granulated), biochemical remission status (remission vs. not in remission), and between T2 hypo- and hyperintense tumours (as assessed through visual inspection) using either a 2-tailed $t$ test or the Mann-Whitney $\mathrm{U}$ test for non-parametric data. Differences in categorical variables (gender and Knosp grade) and remission rate post trans-sphenoidal surgery between intermediate/densely and sparsely granulated somatotropinomas and between T2 hypo- and hyperintense tumours were assessed using Fisher's exact test.

The ability of each MR parameter (including tumour size) to predict tumour functional status and somatotropinoma subtype was assessed through logistic regression analysis and calculation of the area under the receiver operator characteristic curve (AUROC). Statistical differences between ROC curves were evaluated using the DeLong's test for 2-correlated ROC curves [29] (Stata package roccomp). The MR parameter with the highest AUROC value for predicting histological type or somatotropinoma subtype was then chosen and the optimal threshold value for this imaging metric was then derived using Youden's index (Sensitivity + Specificity - 1) [30]. Pretreatment predictors of somatropinoma biochemical remission 
Table 1. Patient demographics and baseline characteristics across non-functioning/functioning tumour groups

\begin{tabular}{|c|c|c|c|c|c|c|}
\hline Median age, years (IQR) & $61.2(53-69)$ & \multicolumn{2}{|c|}{$44.1(32-56)$} & \multicolumn{2}{|c|}{$42.9(35-57)$} & $<0.001$ \\
\hline Male & 44 & \multicolumn{2}{|l|}{11} & \multicolumn{2}{|l|}{19} & \multirow{2}{*}{$0.24^{*}$} \\
\hline Female & 23 & \multicolumn{2}{|c|}{5} & \multicolumn{2}{|c|}{19} & \\
\hline Median tumour diameter, $\mathrm{cm}$ (IQR) & $2.31(1.7-3.0)$ & \multicolumn{2}{|c|}{$2.95(2.3-3.9)$} & \multicolumn{2}{|c|}{$1.11(0.9-1.9)$} & $<0.001$ \\
\hline \multirow{4}{*}{ Knosp grade, $N$} & 13 & I & 1 & I & 8 & \\
\hline & 17 & II & 5 & II & 6 & \multirow[t]{3}{*}{$<0.001^{*}$} \\
\hline & 24 & III & 1 & III & 3 & \\
\hline & IV $\quad 11$ & IV & 9 & & 4 & \\
\hline Median serum prolactin mU/L (IQR) & $341(167-477)$ & 30 & $400-90,000)$ & 214 & 505) & $<0.001$ \\
\hline
\end{tabular}

GH, growth hormone (somatotropinoma); NFG, non-functioning gonadotropinoma; PRL, macroprolactinoma; SI, signal intensity. Tumour diameter $=$ maximum diameter of tumour on midsagittal coronal T2-weighted image. ${ }^{*} p$ value calculated using the KruskalWallis test. For differences in patient gender and Knosp grade, $p$ value determined using Fisher's exact test.

post trans-sphenoidal surgery were similarly assessed through use of an AUROC analysis and multiple logistic regressions with Knosp grade, tumour subtype, biochemical data, and quantitative MR parameters as independent predictors. Bland-Altman analysis [31] was used to assess the agreement between the whole tumour and ROIderived mean SI values. For assessing the intra-observer repeatability of volumetric tumour measurements, Bland-Altman analysis and the intraclass correlation coefficient were used.

\section{Results}

Patient Demographics and Histological Subtype

A CONSORT diagram detailing included/excluded patients is shown in online suppl. Figure 1; see www.karger.com/doi/10.1159/000516823 for all online suppl. material. Sixty-seven patients with NFG, 38 patients with somatotropinoma and 16 patients with macroprolactinoma were included in this study (total $N=121$ ). Seven patients had sparsely granulated somatotropinoma, and $31 \mathrm{pa}-$ tients had either intermediate (6 patients) or densely granulated tumours (25 patients). In somatotropinomas $(N=38)$, biochemical remission post-surgery was achieved in $26(68 \%)$ and $12(32 \%)$ patients did not achieve remission according to the 2010 consensus criteria.

Patient demographics and baseline characteristics across non-functioning/functioning tumour groups are shown in Table 1. Patients with NFG were older than patients with macroprolactinoma $(p=0.001)$ and somatotropinoma (Kruskal-Wallis test, $p<0.001$ ). NFG was larger than somatotropinomas, but the difference was not statistically significant ( $p=0.11$, Kruskal-Wallis test). All included prolactinomas were $>1 \mathrm{~cm}$ in maximum diameter and displayed significantly larger volumes than either NFG ( $p=0.001)$ or somatotropinomas (KruskalWallis test, $p<0.001)$. Compared to excluded tumours with a prolactin level $<10,000 \mathrm{mU} / \mathrm{L}(n=12)$, patients within the included macroprolactinoma cohort were older (Mann-Whitney U test, $p<0.001$ ), predominantly male (Fisher's exact test, $p=0.27$ ), and displayed greater tumour size (Mann-Whitney U test, $p<0.001$ ) and higher tumour invasiveness as measured through Knosp grade (Fisher's exact test, $p<0.001$, online suppl. Table $1)$.

\section{Gonadotroph, Somatotrotroph, and}

Macroprolactinoma Types

As shown in Table 2, derivation of relative SI ratios using both reference regions demonstrated significantly lower tumour/WM and tumour/CSF T2W SI ratio values in somatotropinomas compared to both NFG (ANOVA, $p<0.001$ ) and macroprolactinomas (ANOVA, $p<0.001$, Fig. 1a). Somatotropinomas also displayed higher relative Tumour/CSF T1W SI ratio values compared to NFG (ANOVA, $p<0.001)$ and macroprolactinomas $(p<0.001$; Table 2). Comparison of mean relative tumour T2/T1 SI ratio values demonstrated that somatotropinomas displayed significantly lower T2/T1 ratios than NFG or macroprolactinomas (ANOVA, $p<0.001$; Fig. 1b). Compared to NFG, macroprolactinomas demonstrated lower tu- 
Table 2. Inter-tumour comparison of quantitative MR parameters across non-functioning and functioning tumours

\begin{tabular}{|c|c|c|c|c|c|c|}
\hline $\begin{array}{l}\text { Tumour MR parameter within } \\
\text { group mean (SD) }\end{array}$ & $\begin{array}{l}\text { NFG } \\
(N=67)\end{array}$ & $\begin{array}{l}\text { PRL } \\
(N=16)\end{array}$ & $\begin{array}{l}\mathrm{GH} \\
(N=38)\end{array}$ & $\begin{array}{l}\text { NFG versus } \\
\text { PR, } p \text { value }\end{array}$ & $\begin{array}{l}\text { NFG versus } \\
\mathrm{GH}, p \text { value }\end{array}$ & $\begin{array}{l}\text { PRL versus } \\
\mathrm{GH}, p \text { value }\end{array}$ \\
\hline Tumour/WM T2W SI ratio & $1.55(0.3)$ & $1.46(0.3)$ & $1.08(0.3)$ & 0.74 & $<0.001$ & $<0.001$ \\
\hline Tumour/WM T1W SI ratio & $0.98(0.1)$ & $0.93(0.1)$ & $1.01(0.1)$ & 0.47 & 0.58 & 0.14 \\
\hline Tumour/CSF T2W SI ratio & $0.56(0.1)$ & $0.53(0.2)$ & $0.37(0.1)$ & 0.98 & $<0.001$ & $<0.001$ \\
\hline Tumour T2/T1 SI ${ }_{\text {Ratio-CSF }}$ & $0.26(0.06)$ & $0.29(0.2)$ & $0.15(0.04)$ & 0.60 & $<0.001$ & $<0.001$ \\
\hline
\end{tabular}

$\mathrm{GH}$, growth hormone (somatotropinoma); NFG, non-functioning gonadotropinoma; PRL, macroprolactinoma; SI, signal intensity. $p$ value calculated using one-way ANOVA., Post hoc analysis of pairwise comparisons was performed using the Bonferroni method.

Fig. 1. Comparison of quantitative MR parameters against tumour functional status. a Boxplot comparison of mean tumour/ WM T2W SI ratio against tumour functional status. ${ }^{*} p \leq 0.05{ }^{* *} p \leq 0.01^{* * *} p \leq$ 0.001. b Boxplot comparison of mean relative tumour T2/T1 SI ratio normalized to centrum semiovale WM (T2/T1 SI RatiowM). c Pretreatment coronal T1W and T2W MR images from 64-year-old patient with a non-functioning gonadotropinoma (Top); 53-year-old patient with a macroprolactinoma (Middle); 35-year-old acromegalic patient with a densely granulated somatotropinoma (Bottom). All patients imaged using a Phillips Intera at 1.5 T. Top left image: ROIs shown within tumour (red), bilateral centrum semiovale (green) and CSF of lateral ventricles (blue). GH, growth hormone (somatotropinoma); NFG, non-functioning gonadotropinoma; PRL, macroprolactinoma; SI, signal intensity; WM, white matter; MR, magnetic resonance; ROI, region of interest.

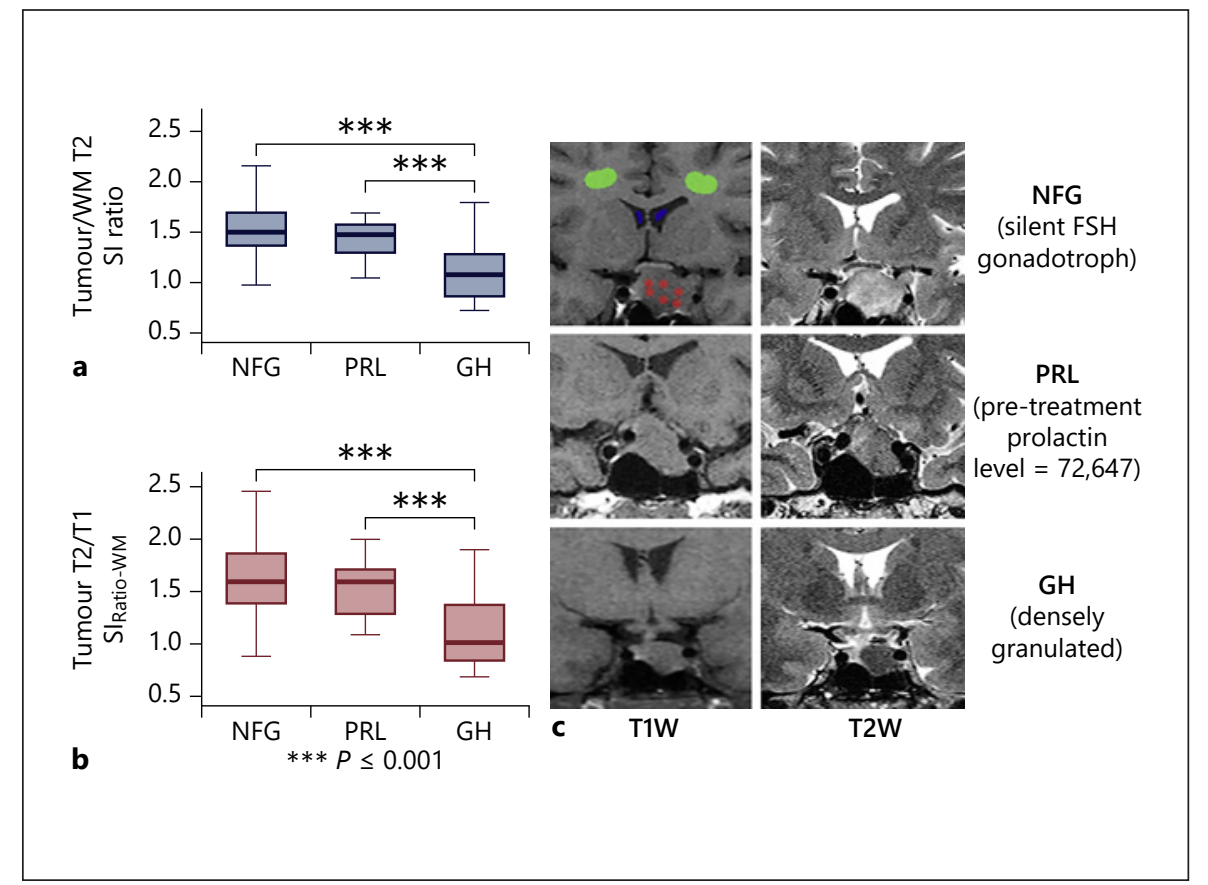

mour/CSF T1W SI ratio values (95\% CI mean difference $0.04,0.31$, ANOVA, $p=0.11$ ) and a non-significant decrease in tumour/WM and tumour/CSF T2W SI ratio values (ANOVA, $p>0.05$ ). Representative coronal T1W and T2W MR images of NFG, macroprolactinoma, and somatotropinomas tumour subtypes are shown in Figure 1c.

\section{Densely versus Sparsely Granulated \\ Somatotropinomas}

The results of subtype analysis are shown in Tables 3 and 4 . Sparsely granulated tumours were significantly larger than intermediate/densely granulated tumours
(Mann-Whitney U test, $p=0.006)$ and demonstrated significantly higher Knosp grade (Fisher's exact test, $p=$ 0.03 , Table 3). Pre-op IGF-1 and GH nadir levels were comparable across the 2 tumour groups (Table 3 ). Sparsely granulated tumours had significantly higher relative T2W SI values (2-tailed $t$ test, $p \leq 0.005$, Fig. $2 \mathrm{a}$ ), and higher relative tumour T2/T1 SI ratio values (2-tailed $t$ test, $p \leq 0.001$, Fig. $2 b)$ than the intermediate/densely granulated group. Representative case examples of coronal T1W and T2W MR image sections of a sparsely and densely granulated somatotropinoma are shown in Figure $2 c$. 
Table 3. Patient demographics and baseline characteristics across somatotropinoma subtypes $(N=38)$

\begin{tabular}{|c|c|c|c|c|c|c|}
\hline \multicolumn{7}{|l|}{ Sex, $N$} \\
\hline Female & 4 & 15 & $0.99^{*}$ & 6 & 13 & \multirow[t]{2}{*}{$0.99^{*}$} \\
\hline Male & 3 & 16 & & 6 & 13 & \\
\hline \multirow[t]{5}{*}{ Knosp grade, $N$} & $0 \quad 1$ & $0 \quad 16$ & $0.03^{*}$ & $0 \quad 2$ & $0 \quad 15$ & \multirow[t]{5}{*}{$0.004^{*}$} \\
\hline & 1 & 7 & & 3 & 5 & \\
\hline & II & II & & II & II & \\
\hline & III & III & & III & III & \\
\hline & IV 3 & IV 1 & & IV & IV & \\
\hline
\end{tabular}

GH, growth hormone (somatotropinoma); SI, signal intensity; TSS, trans-sphenoidal surgery; ULN, upper limit of normal. Tumour diameter = maximum diameter of tumour on midsagittal coronal T2-weighted image. ${ }^{*} p$ value calculated using Mann-Whitney U test. For differences in patient gender and Knosp grade, $p$ value determined using Fisher's exact test.

Table 4. For somatotropinomas, inter-tumour comparison of quantitative MR parameters by granulation status $(N=38)$

\begin{tabular}{lllc}
\hline MR parameter within group mean $( \pm \mathrm{SD})$ & $\begin{array}{l}\text { Sparsely granulated GH } \\
(N=7)\end{array}$ & $\begin{array}{l}\text { Densely/intermediate } \\
\text { granulated GH }(N=31)\end{array}$ & $p$ value \\
\hline Tumour/WM T2W SI ratio & $1.31(0.1)$ & $1.03(0.2)$ & 0.005 \\
Tumour/WM T1W SI ratio & $0.92(0.1)$ & $1.03(0.1)$ & 0.05 \\
Tumour/CSF T2W SI ratio & $0.47(0.1)$ & $0.34(0.1)$ & $<0.001$ \\
Tumour/CSF T1W SI ratio & $2.25(0.3)$ & $2.55(0.4)$ & 0.07 \\
Tumour T2/T1 SI Ratio-WM & $1.44(0.19)$ & $1.02(0.29)$ & 0.001 \\
Tumour T2/T1 SI Ratio-CSF & $0.21(0.03)$ & $0.14(0.03)$ & $<0.001$ \\
\hline
\end{tabular}

SI, signal intensity; WM, white matter; MR, magnetic resonance. $p$ value calculated using 2-tailed $t$ test.

As shown in online suppl. Table 2, tumours judged to be hyperintense on T2W MR imaging were larger (MannWhitney $\mathrm{U}$ test, $p=0.02$ ), demonstrated significantly higher relative T2W SI values (2-tailed $t$ test, $p<0.001$ ), and higher relative tumour T2/T1 SI ratio values (2-tailed $t$ test, $p<0.001)$ than the T2 hypointense tumours. Granulation pattern was significantly different between both groups (Fisher's exact test, $p<0.001$, online suppl. Table 2 ), and all T2 hypointense somatotropinoma in our cohort were intermediate/densely granulated.

Differences in tumour size and relative SI ratios between NFG/macroprolactinomas and each somatotropinoma subtype are shown in online suppl. Table 3. In line with the results for the whole somatotropinoma cohort, densely/intermediate granulated somatotropinomas demonstrated significantly lower relative T2W SI values compared to non-functioning tumours (ANOVA, $p<$ 0.001 ) and macroprolactinomas (ANOVA, $p<0.001$, online suppl. Table 3). Sparsely granulated somatotropinomas also demonstrated lower tumour/WM and tumour/ CSF T2W SI ratio values and higher tumour/CSF T1W SI ratio than both NFG and prolactinomas, but these differences were not statistically significant (ANOVA, $p>0.05$ ).

\section{Post-Operative Remission in Patients with}

Somatotropinoma

Biochemical remission was achieved post-surgery in 26/38 (68\%) patients with somatotropinomas. Seven 
Table 5. For somatotropinomas, inter-tumour comparison of quantitative MR parameters and granulation status by post-surgical resection remission status $(N=38)$

\begin{tabular}{|c|c|c|c|c|c|}
\hline $\begin{array}{l}\text { MR parameter within group mean } \\
( \pm S D)\end{array}$ & $\begin{array}{l}\text { No biochemical remiss } \\
\text { post TSS }(N=12)\end{array}$ & & $\begin{array}{l}\text { Biochemical remission } \\
\text { post TSS }(N=26)\end{array}$ & & $\begin{array}{l}p \\
\text { value }\end{array}$ \\
\hline Tumour/WM T2W SI ratio & $1.13(0.2)$ & & $1.06(0.3)$ & & 0.39 \\
\hline Tumour/WM T1W SI ratio & $0.94(0.1)$ & & $1.04(0.1)$ & & 0.03 \\
\hline Tumour/CSF T2W SI ratio & $0.36(0.1)$ & & $0.37(0.1)$ & & 0.82 \\
\hline Tumour/CSF T1W SI ratio & $2.38(0.3)$ & & $2.55(0.4)$ & & 0.22 \\
\hline Tumour T2/T1 SI Ratio-WM & $1.22(0.29)$ & & $1.04(0.32)$ & & 0.10 \\
\hline Tumour T2/T1 SI Ratio-CSF & $0.16(0.05)$ & & $0.15(0.04)$ & & 0.50 \\
\hline \multirow[t]{2}{*}{ Tumour granulation status, $n(\%)$} & Sparsely & $4(33)$ & Sparsely & $3(12)$ & \multirow{2}{*}{$0.12^{*}$} \\
\hline & Densely/intermediate & $8(66)$ & Densely/intermediate & $23(88)$ & \\
\hline
\end{tabular}

SI, signal intensity; TSS, Trans-sphenoidal surgery; MR, magnetic resonance. ${ }^{*} p$ value calculated using 2 -tailed $\mathrm{t}$ test. For differences in tumour granulation status $p$ value determined using Fisher's exact test.

Fig. 2. Somatotroph granulation status. a Boxplot comparison of pretreatment mean tumour/WM T2W SI ratio against somatotropinoma granulation status. b Boxplot comparison of pretreatment mean relative tumour T2/T1 SI ratio normalized to CSF (T2/T1 SI $I_{\text {Ratio-CSF }}$ ). c Representative T1W and T2W coronal image sections in a 26-year-old patient with a sparsely granulated tumour (left); and a 57-year-old patient with a densely granulated tumour (right, *). Both patients imaged with a GE Optima MR450W at 1.5 T. Data shown for 7 sparsely and 31 intermediate/densely granulated somatotropinomas. SI, signal intensity; WM, white matter.

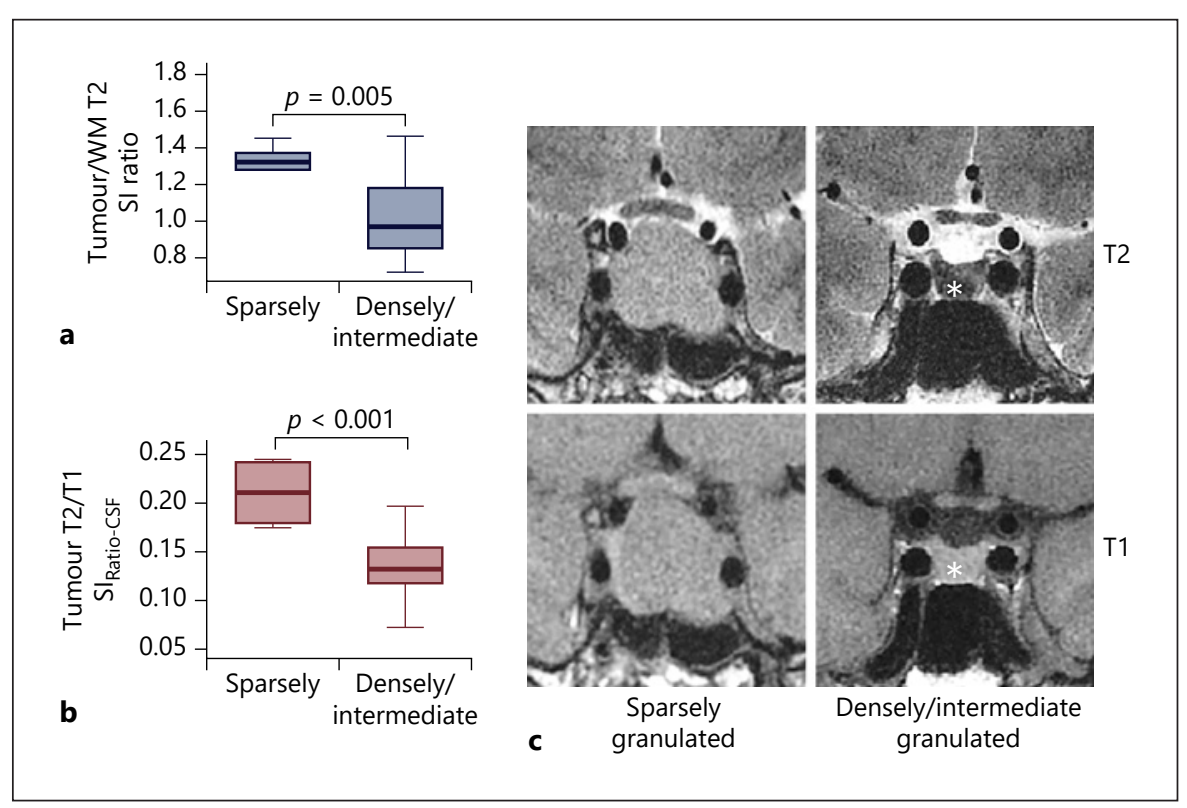

(18\%) were not in remission, including 3 patients who had undergone debulking and a further 3 patients in whom complete resection was planned but intra-operatively tumour spread into the cavernous sinus and/or bony clivus was discovered. Five (13\%) patients had borderline results and thus as per the 2010 consensus guidelines, they were included in the "not in remission" group. In 23 patients, remission was achieved following the first trans-sphenoidal surgery and 3 patients went into remission following a second surgical procedure.

Patients who achieved biochemical remission had significantly lower Knosp grade (Fisher's exact test, $p=$
0.004, Table 3), smaller tumours at diagnosis (MannWhitney U test, $p=0.003$, Table 3), and higher tumour/ WM T1W SI values (2-tailed $t$ test, $p=0.03$, Table 5 ) than patients not in remission. Those patients with somatotropinomas achieving biochemical remission trended to have densely/intermediate granulated tumours than patients not in remission, although this failed to be significant (Fisher's exact test, $p=0.12$ ).

\section{AUROC Characteristics}

Comparison of the AUROC for each quantitative MR parameter is shown in online suppl. Table 4 . For differ- 
Table 6. AUROC, sensitivity, and specificity of individual quantitative MR parameters for the prediction of adenohypophyseal tumour functional/granulation status

\begin{tabular}{|c|c|c|c|c|c|c|c|}
\hline & Quantitative MR parameter & $\begin{array}{l}\text { AUROC (95\% } \\
\text { confidence interval) }\end{array}$ & $\begin{array}{l}\text { Optimal threshold } \\
\text { value }\end{array}$ & $\begin{array}{l}\text { Sensitivity, } \\
\%\end{array}$ & $\begin{array}{l}\text { Specificity, } \\
\%\end{array}$ & $\begin{array}{l}\text { Correctly } \\
\text { classified, \% }\end{array}$ & $\begin{array}{l}\text { Youden's } \\
\text { index }(\mathrm{J})\end{array}$ \\
\hline NFG versus GH & Tumour T2/T1SI Ratio-CSF & $0.94(0.89-0.98)$ & $\geq 0.20$ & 91.0 & 86.8 & 89.5 & 0.78 \\
\hline NFG versus PRL & Tumour/CSF T1W ratio & $0.67(0.50-0.83)$ & $\geq 2.04$ & 80.6 & 56.3 & 75.9 & 0.37 \\
\hline PRL versus GH & Tumour T2/T1SI $I_{\text {Ratio-CSF }}$ & $0.90(0.81-0.99)$ & $\geq 0.22$ & 75.0 & 89.5 & 85.2 & 0.65 \\
\hline $\begin{array}{l}\text { Sparsely versus densely/ } \\
\text { intermediate GH }\end{array}$ & Tumour T2/T1SI Ratio-CSF & $0.95(0.88-1.00)$ & $\geq 0.18$ & 100.0 & 87.1 & 89.5 & 0.87 \\
\hline
\end{tabular}

GH, growth hormone (somatotropinoma); NFG, non-functioning gonadotropinoma; PRL, macroprolactinoma; SI, signal intensity; AUROC, area under the receiver operator characteristic curve; MR, magnetic resonance. Optimal cut-off point for each parameter chosen using the Youden's index (J). Sensitivity, specificity, and \% correctly classified shown.

entiating NFG from functioning (macroprolactinoma/ somatotropinoma) tumours, relative T2W SI ratio values (AUROC $=0.80-0.82$ ), and the tumour T2/T1 SI Ratio-CSF $($ AUROC $=0.81)$ displayed significantly higher AUROC values than either tumour volume (AUROC $=0.67, p \leq$ 0.01 ) or Knosp grade (AUROC $=0.63, p<0.002$ ). Subgroup analysis on 37 patients imaged using the same scanner system (Phillips Intera) demonstrated increased AUROC values for relative T2W SI ratio values (AUROC $=0.83-0.89)$ and relative tumour $\mathrm{T} 2 / \mathrm{T} 1$ SI ratio values (AUROC $=0.83-0.84$ ) in differentiating NFG from functioning tumours.

As shown in online suppl. Table 5, relative tumour T2W SI values (AUROC $=0.87-0.94)$ and relative tumour T2/T1 ratio values $($ AUROC $=0.89-0.95)$ demonstrated good discriminatory power in differentiating somatotropinoma granulation status. There was no statistically significant difference in the estimated AUROC values across the different MR imaging metrics including tumour size and Knosp grade $(p>0.05)$. Compared to pre-op IGF-1 (AUROC $=0.73, p=0.03)$ and pre-op GH nadir (AUROC $=0.51, p<0.001$ ), the tumour T2/T1 SI

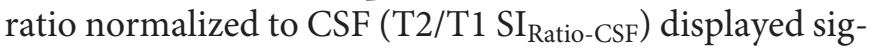
nificantly higher AUROC for differentiating somatotropinoma granulation status. As shown in Table 6, at a threshold of 0.18 the tumour T2/T1 SI Ratio-CSF $_{\text {had } 100 \%}$ sensitivity and $87.1 \%$ specificity for differentiating sparsely from intermediate/densely granulated somatotropinomas. For predicting remission post trans-sphenoidal surgery (online suppl. Table 5), tumour size (AUROC = $0.80)$ and Knosp grade $($ AUROC $=0.79)$ had higher $(p>$ $0.05)$ AUROC values than all relative tumour SI measures (AUROC $=0.51-0.73$ ), but these differences were not statistically significant.

Pre-Treatment MRI Biomarkers of Adenohypophyseal Tumour Phenotype

\section{ROI and Whole Tumour Based Methods}

The results of Bland-Altman analysis comparing the mean absolute T2W and T1W SI values derived using both the whole tumour perimetry approach and the multiple ROI approach are shown in online suppl. Figure 2. The overall limit of agreement using the 2 methods was $\pm 20 \%$, and use of the ROI approach generated systematically higher absolute T2W (mean difference $=-2.4 \%$ ) and $\mathrm{T} 1 \mathrm{~W}$ values (mean difference $=-0.8 \%$ ). Test-retest analysis of tumour volume using manual delineation is shown in online suppl. Figure 2c. Whilst the second tumour volume estimate was systematically higher (mean difference $=-2.9 \%$ ), this percentage difference decreased with increasing tumour size, and there was overall good repeatability with a single-measure absolute difference ICC of 0.99 .

\section{Discussion}

In this retrospective exploratory cohort study, we have demonstrated that non-functioning and functioning anterior adenohypophyseal tumours differ in their quantitative T2W SI characteristics and that these differences can help differentiate between tumour subtypes. Normalized relative SI ratios demonstrated that somatotropinomas notably densely granulated subtype display lower T2W SI values than NFG and a cohort of macroprolactinomas.

Lower T2W SI values in somatotropinomas support the results of earlier studies [7, 10, 12, 13]. Different theories have been formulated to explain such differences [12, 13]. Hormone secretion and subsequent shortening of the $\mathrm{T} 2$ relaxation rates of nearby water protons was proposed to cause the lower T2W SI seen 
in functioning compared to non-functioning tumours $[7,13,32]$. Microscopic cysts and areas of necrosis can occur in adenohypophyseal tumours. Smaller areas of microscopic necrosis in somatotropinoma compared to NFG and prolactinoma may also partly explain their comparatively lower T2W SI values [12, 33, 34]. By contrast, previous studies have suggested that treatmentnaïve prolactinomas and NFG display similar intratumoural necrosis $[12,33]$, a finding that may explain the absence of significant quantitative T2W SI differences between these 2 tumours in our study. Data in the literature on the correlation between fibrosis and T2W SI are contradictory, reporting either no $[8,35,36]$ or inverse correlation [37-39].

The ability of our quantitative T1W and T2W MR parameters to predict the histotype of adenohypophyseal tumours may have some application in their management. Our study demonstrated that the use of a quick, multiple ROI-based tumour sampling method offers comparable results, and it is conceivable that such a method could be incorporated into MR image analysis software to aid in pretreatment differentiation of adenohypophyseal tumours. One such application could be to help differentiate NFG with raised prolactin levels due to stalk effect from true prolactinomas. Due to a limited number of surgically resected prolactinomas at our centre, a cohort of medically treated, giant macroprolactinomas that displayed high pretreatment prolactin levels $(>10,000 \mathrm{mU} / \mathrm{L})$ and clear biochemical/radiological response to treatment were included. These observations will therefore require further larger studies with inclusion of a group of smaller prolactinomas with moderate prolactin levels before these findings can be generalized to the wider prolactinoma cohort.

In this quantitative study, we observed that densely granulated somatotropinoma display lower SI values on T2W imaging than sparsely granulated tumours, findings which are consistent with the previous qualitative studies $[7,10,11,40]$. Increased hormone secretion into the tumoural capillary network [41] and an increased abundance of secretory granules in densely granulated somatotropinomas have been previously proposed as reasons behind this feature $[13,40,42]$. In previous studies, it has been shown that sparsely granulated somatotropinomas show less biochemical response to somatostatin analogues compared to densely granulated tumours [23, 40, 43]. Our demonstration that quantitative T2W SI measures can differentiate the subtype of pretreatment somatotropinomas may therefore be of clinical use to stratify somatotropinoma histological subtypes and thus identify patients who would benefit from preoperative medical treatment [44]. This lends further support to previous studies which have demonstrated a correlation between tumour T2W SI values at baseline and tumour shrinkage with somatostatin analogues $[11,45]$. In this study, all patients with GH-secreting tumours underwent trans-sphenoidal surgery as their primary treatment and biochemical remission rates following surgery (68\%) are in line with recent publications from specialist pituitary centres [25]. Tumour size, extent of tumour invasion, and surgeon experience have all been shown to impact on remission rates post-surgery $[25,46]$, and in this single-surgeon study, both higher Knosp grade [27] and larger tumour volume were associated with failure to achieve biochemical remission post-surgery. Whilst sparsely granulated somatotropinomas did demonstrate a trend to lower remission rates, these tumours were also larger.

To our knowledge, this is one of the largest studies investigating quantitative MR parameters across different adenohypophyseal tumour types and subtypes. Due to its retrospective nature, however, the study did suffer from some limitations. Thus, patients with limited and/or inadequate imaging were excluded from this study. In addition, we only investigated somatotropinomas and NFG that were surgically resected, focusing the study on larger, non-functioning tumours, and somatotropinomas that were suitable for surgical resection rather than medical therapy. Tumours with significant cystic/haemorrhagic components were excluded to reduce confounding features on the measured MR signal intensities. In the present study, patients had been imaged using 5 different MR scanner vendors, at different times, using different MR scanner protocols and use of relative SI measures obtained from a single scanner improved differentiation between different pituitary subtypes. Larger prospective studies using the same scanner vendor and fixed acquisition are therefore required to better verify our study findings.

\section{Conclusion}

This quantitative MR study demonstrated that nonfunctioning and functioning adenohypophyseal tumours differ in their quantitative T1W and T2W SI characteristics on pretreatment MRI and that these SI differences can be used as potential markers to help non-invasively predict the functional status and certain histotypes. 


\section{Acknowledgements}

The authors thank all the staff within the research and development division at Salford Royal Hospital for their invaluable help during the ethical approval application for the study. We also thank the wider pituitary team in the region for sharing the clinical data on these patients.

\section{Statement of Ethics}

The study was approved by the East of England Research ethics committee (REC Reference, 17/EE/0488). All study procedures were performed in accordance with the ethical standards of the institutional and national research committees and in accordance with the 1964 Helsinki Declaration and its later amendments.

\section{Conflict of Interest Statement}

The authors have no conflicts of interest to declare.

\section{Funding Sources}

The work was supported by funding from the CRUK Cancer Imaging Centre in Cambridge and Manchester (Grant number: C8742/A18097). D.L. is supported by a National Institute for Health Research (NIHR) Academic Clinical Fellowship.

\section{Author Contributions}

D.L. and K.G. conceived the study. D.L., F.R., T.K., and K.G. collected the data. D.L. and D.C. performed the data analysis. D.L., F.R., T.K., D.C., and K.G. edited the manuscript, and all the authors have read and approved the manuscript.

\section{References}

1 Freda PU, Post KD. Differential diagnosis of sellar masses. Endocrinol Metab Clin North Am. 1999;28(1):81-vi.

2 Lopes MBS. The 2017 World Health Organization classification of tumors of the pituitary gland: a summary. Acta Neuropathol. 2017 Oct:134(4):521-35.

3 Pisaneschi M, Kapoor G. Imaging the sella and parasellar region. Neuroimaging Clin North Am. 2005;15(1):203-19.

4 Biller BM, Colao A, Petersenn S, Bonert VS, Boscaro M. Prolactinomas, Cushing's disease and acromegaly: debating the role of medical therapy for secretory pituitary adenomas. BMC Endocr Disord. 2010;10:10.

5 Greenman Y, Stern N. Non-functioning pituitary adenomas. Best Pract Res Clin Endocrinol Metab. 2009;23(5):625-38.

6 Marro B, Zouaoui A, Sahel M, Crozat N, Gerber S, Sourour N, et al. MRI of pituitary adenomas in acromegaly. Neuroradiology. 1997; 39(6):394-9.

7 Hagiwara A, Inoue Y, Wakasa K, Haba T, Tashiro T, Miyamoto T. Comparison of growth hormone-producing and non-growth hormone-producing pituitary adenomas: imaging characteristics and pathologic correlation. Radiology. 2003;228(2):533-8.

8 Chakrabortty S, Oi S, Yamaguchi M, Tamaki N, Matsumoto S. Growth hormone-producing pituitary adenomas: MR characteristics and pre- and postoperative evaluation. Neurol Med Chir. 1993;33(2):81-5.

9 Lazaro CM, Guo WY, Sami M, Hindmarsh T, Ericson K, Hulting AL, et al. Haemorrhagic pituitary tumours. Neuroradiology. 1994 Feb; 36(2):111-4.

10 Dogansen SC, Yalin GY, Tanrikulu S, Tekin S, Nizam N, Bilgic B, et al. Clinicopathological significance of baseline T2-weighted signal intensity in functional pituitary adenomas. Pituitary. 2018 Aug;21(4):347-54.
11 Heck A, Emblem KE, Casar-Borota O, Bollerslev J, Ringstad G. Quantitative analyses of T2-weighted MRI as a potential marker for response to somatostatin analogs in newly diagnosed acromegaly. Endocrine. 2016 May; 52(2):333-43.

12 Lundin P, Nyman R, Burman P, Lundberg PO, Muhr C. MRI of pituitary macroadenomas with reference to hormonal activity. Neuroradiology. 1992;34(1):43-51

13 Yiping L, Ji X, Daoying G, Bo Y. Prediction of the consistency of pituitary adenoma: a comparative study on diffusion-weighted imaging and pathological results. J Neuroradiol. 2016 Jun;43(3):186-94.

14 Drummond J, Roncaroli F, Grossman AB, Korbonits M. Clinical and pathological aspects of silent pituitary adenomas. J Clin Endocrinol Metab. 2019 Mar;104(7):2473-89.

15 Obari A, Sano T, Ohyama K, Kudo E, Qian ZR, Yoneda A, et al. Clinicopathological features of growth hormone-producing pituitary adenomas: difference among various types defined by cytokeratin distribution pattern including a transitional form. Endocr Pathol. 2008;19(2):82-91.

16 Lonser RR, Nieman L, Oldfield EH. Cushing's disease: pathobiology, diagnosis, and management. J Neurosurg. 2017 Feb;126(2):40417.

17 Sied JG, Zijdenbos AP, Evans AC. A nonparametric method for automatic correction of intensity nonuniformity in mri data. IEEE Trans Med Imaging. 1998;17(1):87-97.

18 Ashburner J, Friston K. Multimodal image coregistration and partitioning: a unified framework. Neuroimage. 1997 Oct;6(3):209_ 17.
19 Yushkevich PA, Piven J, Hazlett HC, Smith RG, Ho S, Gee JC, et al. User-guided 3D active contour segmentation of anatomical structures: significantly improved efficiency and reliability. Neuroimage. 2006;31(3):1116-28.

20 Davies BM, Carr E, Soh C, Gnanalingham KK. Assessing size of pituitary adenomas: a comparison of qualitative and quantitative methods on MR. Acta Neurochir. 2016; 158(4):677-83

21 Doubal FN, MacLullich AM, Ferguson KJ, Dennis MS, Wardlaw JM. Enlarged perivascular spaces on MRI are a feature of cerebral small vessel disease. Stroke. 2010 Mar;41(3): $450-4$.

22 Sherman JL, Citrin CM. Magnetic resonance demonstration of normal CSF flow. AJNR Am J Neuroradiol. 1986;7(1):3-6.

23 Bhayana S, Booth GL, Asa SL, Kovacs K, Ezzat $S$. The implication of somatotroph adenoma phenotype to somatostatin analog responsiveness in acromegaly. J Clin Endocrinol Metab. 2005;90(11):6290-5.

24 Reid TJ, Jin Z, Shen W, Reyes-Vidal CM, Fernandez JC, Bruce JN, et al. IGF-1 levels across the spectrum of normal to elevated in acromegaly: relationship to insulin sensitivity, markers of cardiovascular risk and body composition. Pituitary. 2015 Dec;18(6):808-19.

25 Wang YY, Higham C, Kearney T, Davis JRE, Trainer P, Gnanalingham KK. Acromegaly surgery in Manchester revisited: the impact of reducing surgeon numbers and the 2010 consensus guidelines for disease remission. Clin Endocrinol. 2012 Mar;76(3):399-406.

26 Leach P, Abou-Zeid AH, Kearney T, Davis J, Trainer PJ, Gnanalingham KK. Endoscopic transsphenoidal pituitary surgery: evidence of an operative learning curve. Neurosurgery. 2010 Nov;67(5):1205-12. 
27 Knosp E, Steiner E, Kitz K, Matula C, Parent $\mathrm{AD}$, Laws ER, et al. Pituitary adenomas with invasion of the cavernous sinus space: a magnetic resonance imaging classification compared with surgical findings. Neurosurgery. 1993;33(4):610-8.

28 Giustina A, Chanson P, Bronstein MD, Klibanski A, Lamberts S, Casanueva FF, et al. A consensus on criteria for cure of acromegaly. J Clin Endocrinol Metab. 2010 Jul;95(7): 3141-8.

29 DeLong ER, DeLong DM, Clarke-Pearson DL. Comparing the areas under two or more correlated receiver operating characteristic curves: a nonparametric approach. Biometrics. 1988 Sep;44(3):837.

30 Youden WJ. Index for rating diagnostic tests. Cancer. 1950;3(1):32-5.

31 Bland JM, Altman DG. Statistical methods for assessing agreement between two methods of clinical measurement. Lancet. 1986;1(8476): 307-10.

32 McRobbie D, Moore EA, Graves MJ, Prince MR. MRI: from picture to proton; 2003.

33 Hallenga B, Saeger W, Lüdecke DK. Necroses of prolactin-secreting pituitary adenomas under treatment with dopamine agonists: light microscopical and morphometric studies. Exp Clin Endocrinol. 1988 Jul;92(4):59-68.

34 Bonneville JF. Magnetic resonance imaging of pituitary tumors. Front Horm Res. 2016;45: 97-120.
35 Alimohamadi M, Sanjari R, Mortazavi A, Shirani M, Moradi Tabriz H, Hadizadeh Kharazi $\mathrm{H}$, et al. Predictive value of diffusion-weighted MRI for tumor consistency and resection rate of nonfunctional pituitary macroadenomas. Acta Neurochir. 2014 Dec;156(12): 2245-52.

36 Mahmoud OM, Tominaga A, Amatya VJ, Ohtaki M, Sugiyama K, Saito T, et al. Role of PROPELLER diffusion weighted imaging and apparent diffusion coefficient in the diagnosis of sellar and parasellar lesions. Eur J Radiol. 2010;74(3):420-7.

37 Pierallini A, Caramia F, Falcone C, Tinelli E, Paonessa A, Ciddio AB, et al. Pituitary macroadenomas: preoperative evaluation of consistency with diffusion-weighted MR imaging: initial experience. Radiology. 2006; 239(1):223-31.

38 Iuchi T, Saeki N, Tanaka M, Sunami K, Yamaura A. MRI prediction of fibrous pituitary adenomas. Acta Neurochir. 1998;140(8):77986.

39 Snow RB, Johnson CE, Morgello S, Lavyne $\mathrm{MH}$, Patterson RH. Is magnetic resonance imaging useful in guiding the operative approach to large pituitary tumors? Neurosurgery. 1990;26(5):801-3.

40 Heck A, Ringstad G, Fougner SL, Casar-Borota $\mathrm{O}$, Nome T, Ramm-Pettersen J, et al. Intensity of pituitary adenoma on T2-weighted magnetic resonance imaging predicts the response to octreotide treatment in newly diagnosed acromegaly. Clin Endocrinol. 2012 Jul; 77(1):72-8
41 Yoshitomi M, Ohta K, Kanazawa T, Togo A, Hirashima S, Uemura KI, et al. Three-dimensional ultrastructural analyses of anterior pituitary gland expose spatial relationships between endocrine cell secretory granule localization and capillary distribution. Sci Rep. 2016 Oct; $6: 36019$.

42 Horvath E, Kovacs K. Pathology of acromegaly. Neuroendocrinology. 2006;83:161-5.

43 Bakhtiar Y, Hirano H, Arita K, Yunoue S, Fujio S, Tominaga A, et al. Relationship between cytokeratin staining patterns and clinicopathological features in somatotropinomae. Eur J Endocrinol. 2010;163(4):531-9.

44 Melmed S, Colao A, Barkan A, Molitch M, Grossman AB, Kleinberg D, et al. Guidelines for acromegaly management: an update. Clin Endocrinol Metab. 2009;94:1509-17.

45 Potorac I, Petrossians P, Daly AF, Alexopoulou O, Borot S, Sahnoun-Fathallah M, et al. T2-weighted MRI signal predicts hormone and tumor responses to somatostatin analogs in acromegaly. Endocr Relat Cancer. 2016 Nov;23(11):871-81.

46 Hofstetter CP, Mannaa RH, Mubita L, Anand VK, Kennedy JW, Dehdashti AR, et al. Endoscopic endonasal transsphenoidal surgery for growth hormone-secreting pituitary adenomas. Neurosurg Focus. 2010 Oct;29(4):E6-7. 